\title{
Aging Mechanisms in RTV Polysiloxane Foams
}

\author{
Andrea Labouriau ${ }^{1 *}$, Tom Robison ${ }^{2}$, Linda Meincke ${ }^{1}$, Debra Wrobleski ${ }^{1}$, Dean Taylor ${ }^{1}$ and \\ John Gill \\ ${ }^{1}$ Los Alamos National Laboratory, Los Alamos, New Mexico, 87545 \\ ${ }^{2}$ Honeywell FM\&T, Kansas City, Missouri, 64147
}

\begin{abstract}
.
The long-term use of polysiloxane foams requires a good understanding of the diverse factors that may influence their performance. The aim of the present work was to understand the interplay between in-service environments and reactive species that reside in the foams. Two foams (SX358 and S5370) were thermally aged under compressive strain to gauge the onset of compression set, an indicator of degradation. Experiments were performed by either aging the foams in open air containers or in high humidity environments. Our results indicated that foams aged in open air environments exhibited less compression set than when aged in presence of moisture. These results were analyzed in terms of post-curing reactions, hydrolysis and rearrangement of the network, which are promoted by active residues that are left in the foam from the curing process. In particular, the residues from tin octoate, the catalyst used in the cure of the foams, were identified and their role in promoting aging was determined. Reactions were either catalyzed by tin(II) species or by a combination of trace amounts of water and octanoic acid, a product of the hydrolysis of tin octoate. More precisely, this work demonstrated that reactive residues promote chemical changes in the polymer, which resulted in compression set. On the other hand, the oxidized residues of the tin octoate show no catalytic activity towards promoting condensation reactions between silanol groups. Our work established that, depending on service conditions, certain residues are more harmful to the foam's lifetime than others.
\end{abstract}

Keywords: Polysiloxanes, hydrolysis, stress relaxation, RTV foams, tin octoate, octanoic acid Corresponding author: A. Labouriau (andrea@lanl.gov), phone: 505-667-7216. 


\section{Introduction.}

Polysiloxane elastomers are extensively used in diverse applications due to their superior properties like good thermal and chemical stability. ${ }^{1}$ Nevertheless, exposure to harsh environmental conditions may adversely impact their long-term performance. For example, it is well known that the stability of polysiloxanes is unfavorably affected by oxygen, water, fillers, traces of acidic or basic impurities, and residual catalyst. ${ }^{2-7}$ Case in point, decomposition products of catalysts are known to affect the stability and mechanical properties of the silicone rubber. $^{8}$ On the other hand, the addition of stabilizers, such as zirconium and cerium 2-ethylhexanoates, has been shown to inhibit thermal degradation. ${ }^{9}$ Therefore, detailed knowledge of aging mechanisms is important not only to predict the lifetime of the material, but also to provide guidance on service conditions to be avoided, and lead to improvements that should be considered when developing replacement materials.

Identifying aging mechanisms that result in changes of the mechanical properties of room temperature vulcanized (RTV) polysiloxane foams was the primary concern. Silica-filled foamed polysiloxanes are engineered as stress-absorbing materials to be used in applications that call for good elastic recovery properties during their lifetime. A set of published studies have shown that these materials undergo compression set when accelerated aged under compressive load. ${ }^{10,11}$ In particular, moisture present in the service environment is likely to react with the polymer producing lower molecular weight siloxanes. ${ }^{12}$ By the same token, residual catalyst is expected to promote post-curing reactions that may increase cross-link density. Generally, changes in the polymer network linkages while the polymer is under strain are associated with permanent set or loss of elastic recovery as has been indicated by several experimental and molecular dynamics studies. ${ }^{13,14}$ Data reported in this work provides valuable insights on the presence of reactive sites and the influence of residual catalyst and its by-products in promoting post-curing reactions, chain scission and bond-exchange reactions. All of these changes are likely to degrade the RTV foam over its lifetime. 


\section{Experimental Section.}

\subsection{Materials.}

Two chemically similar polysiloxane foams, S5370 and its replacement foam known as SX358 were artificially aged under compressive strain.

SX358 foams were newly synthesized from a polysiloxane elastomeric gumstock that contained $75 \mathrm{wt} \%$ dihydroxyl-terminated polydimethylsiloxane (HO-PDMS-OH), 3wt\% polymethylhydrosiloxane (PMHS), 2wt\% tetrapropyl orthosilicate (TPS), and 5wt\% diphenylmethylsilanol (DPMS); all of which were purchased from NuSil Technologies. The dihydroxyl-terminated polydimethylsiloxane consisted of a mixture of about $17 \mathrm{wt} \%$ low molecular weight ( $\left.\mathrm{M}_{\mathrm{w}}=2,350\right), 14.5 \mathrm{wt} \%$ medium molecular weight $\left(\mathrm{M}_{\mathrm{w}}=29,400\right)$, and $43.5 \mathrm{wt} \%$ high molecular weight $\left(M_{w}=54,300\right)$. The uncured resin also contained $15 \mathrm{wt} \%$ of diatomaceous earth filler to provide reinforcement to the cured polymer. SX358 foams were cured by condensation reactions between silanols on dihydroxyl-terminated PDMS, and DPMS and silanes on PMHS, producing hydrogen gas. Condensation between silanols and TPS also contributed to cross-linking, with evolution of $n$-propyl alcohol. Both condensation reactions were catalyzed by tin octoate (also known as tin 2-ethylhexanoate). The procedure consisted of mixing 5 wt\% tin octoate into the polysiloxane gumstock, and allowing it to cure at room temperature into a mold to obtain a chosen thickness (the average thickness of pristine

samples was $0.102 \mathrm{~cm}$ ) and density (about $0.4 \mathrm{~g} / \mathrm{cm}^{3}$ ). This amount of tin catalyst is necessary in order to produce foams of high-quality. The foams were post-cured by heating at $115^{\circ} \mathrm{C}$ in air for 3.5 hours. SX358 samples were artificially aged in air at temperatures of 30, 50, 70, 90 and $110^{\circ} \mathrm{C}$ in convection ovens. There were three specimens per aging condition that were periodically withdrawn to measure changes in thickness. Then, the samples were placed back into the compression fixtures and ovens for continued aging. Stackable compression fixtures were made from aluminum sheet stock with sample wells machined for the appropriate depth to accommodate $5.0 \mathrm{~cm}$ diameter sample discs at $25 \%$ compressive strain. The fixtures used in this work also had two small indents that allowed for air to diffuse through the samples. 
S5370 foams were originally prepared from a commercial kit (Dow Corning Silastic5370) that is no longer available. ${ }^{10}$ These materials were synthesized in 1985 and stored under low humidity conditions at 20 to $22^{\circ} \mathrm{C}$. Thus, S5370 foams were already naturally aged for 28 years before being artificially aged and tested in this work. The reasoning to use naturally aged S5370 foams was to investigate the role of long-lived residues in the long-term aging of RTV foams. S5370 samples had an average thickness of $0.108 \mathrm{~cm}$ and density of $0.4 \mathrm{~g} / \mathrm{cm}^{3}$. Samples were cut into approximately $5 \mathrm{~cm}$ diameter discs and thermally aged at $70^{\circ} \mathrm{C}$, under fixed compressive loading (about 30\%). Specimens were aged for up to 2 years at controlled humidity levels of 10 and 25\% using commercial humidity chambers (ESPEC North America Inc., Michigan, USA). A set of two samples per aging condition were analyzed at 3-months intervals (newly aged samples were never returned to the humidity chambers).

Bond exchange reactions, or rearrangement of the network, were evaluated by probing variations in the molecular weight distribution of tri-methyl-terminated PDMS $\left(M_{n}=10,000\right.$, American Polymer Laboratories) when mixed with $5 \mathrm{wt} \%$ tin octoate (Aldrich-Sigma). The tin catalyst sample was initially analyzed by ${ }^{119} \mathrm{Sn}$ Mössbauer spectroscopy, confirming that it contained mostly tin(II) species (85\% tin(II) and 15\% tin (IV)). For a period of up to 14 weeks, aliquots were periodically taken and investigated by gel permeation chromatography (GPC). Since no significant changes in the PDMS molecular weight or in its polydispersity index $\left(M_{w} / M_{n}\right)$ were observed for the first 8 weeks, the sample was heated to $70^{\circ} \mathrm{C}$ for an additional 6 weeks; aliquots were analyzed by GPC at 3 and 6 weeks.

Acid-catalyzed hydrolysis reactions were investigated by monitoring changes in the molecular weight of tri-methyl-terminated PDMS $\left(M_{w}=97,200, M n=51,900\right.$, Sigma-Aldrich, 200 mg of polymer for each aging condition) when mixed with 5 wt\% of octanoic acid (also known as 2-ethyl-hexanoic acid, Sigma-Aldrich) and $10 \mu$ l of water. These samples were aged at 40, 70, 90 , and $110^{\circ} \mathrm{C}$ for up to 103 days; aliquots were periodically investigated by GPC.

The ability of the tin catalyst for promoting condensation reactions or chain extension reactions was examined by mixing low molecular weight dihydroxyl-terminated PDMS (NuSil Technologies) with $5 \mathrm{wt} \%$ of tin octoate (these were new and degraded batches of the tin 
catalyst). Samples were dissolved in chloroform and readily analyzed by liquid-state ${ }^{29}$ Si NMR spectroscopy (or within one hour after sample preparation). ${ }^{17} \mathrm{O}$ insertion in the polymer backbone was studied by artificially aging post-cured and non-post-cured SX358 foams in excess of isotopically enriched ${ }^{17} \mathrm{O}$ water $\left({ }^{17} \mathrm{O}, 20 \%\right.$ Cambridge Isotope Laboratories, Inc.) in closed containers at $60^{\circ} \mathrm{C}$ for 23 weeks. These samples were analyzed by solid-state ${ }^{17} \mathrm{O}$ NMR spectroscopy.

Residues from the tin catalyst present in RTV foams were evaluated by extracting the samples (380 mg) in deuterated chloroform ( $\mathrm{CDCl}_{3}, 99.6 \%$ Acros Organics) for 4 hours at room temperature. The soluble fraction was about 3 to $5 \%$ of the initial mass of the sample. The extracted material $(0.75 \mathrm{ml})$ was analyzed by liquid-state ${ }^{1} \mathrm{H}$ NMR spectroscopy.

\subsection{Techniques.}

Liquid-state ${ }^{1} \mathrm{H}$ and ${ }^{29} \mathrm{Si}$ NMR experiments were carried out using a Bruker Avance NMR spectrometer operating at $300.13 \mathrm{MHz}$ for ${ }^{1} \mathrm{H}$ and at $59.62 \mathrm{MHz}$ for ${ }^{29} \mathrm{Si}$, proton and silicon signals were referenced to internal tetramethylsilane (TMS). ${ }^{17} \mathrm{O} N M R$ spectroscopy was performed on RTV foams that were artificially aged with excess of ${ }^{17} \mathrm{O}$ isotopically enriched water. ${ }^{17} \mathrm{O} N M R$ experiments were performed using a Bruker solid-state NMR spectrometer operating at $54.24 \mathrm{MHz}$. Samples were spun to $5 \mathrm{kHz}$ using a $4 \mathrm{~mm}$ Magic Angle Spinning (MAS) probe. ${ }^{1} \mathrm{H}$ MAS NMR experiments were performed on pristine SX358 foams using a Bruker Avance NMR spectrometer operating at $400.13 \mathrm{MHz}$ equipped with a Bruker (4 mm) Magic Angle Spinning (MAS) probe. Samples were spun at about $9 \mathrm{KHz} .{ }^{1} \mathrm{H}$ MAS NMR was used to characterize the foam's network, especially to detect unreacted chemical groups such as silanols and silanes.

${ }^{119} \mathrm{Sn}$ Mössbauer spectroscopy was performed at low temperature (75K) using an integral cryostat and spectrometer. A commercial $\mathrm{Ca}^{119 \mathrm{~m}} \mathrm{SnO}_{3}$ source provided the $23.87 \mathrm{keV}$ gamma rays for the Mössbauer spectroscopy. Spectra were fitted using the appropriate 
Hamiltonian and least square-fitting program. The statistical errors associated with the fitting procedure are of the order of $\pm 0.02 \mathrm{~mm} / \mathrm{s}$ in velocity or $0.5 \%$ in transmission. The inherent recoil-free fraction for Sn(IV) is slightly greater than that for $\mathrm{Sn}(\mathrm{II})$, and a correction has been applied to reflect this difference as it applies to determining the $\mathrm{Sn}$ (IV)/Sn(II) relative abundances. $^{15}$

Mass spectroscopy was performed to detect volatile species and permanent gases in the headspace of thermally aged SX358 foam. The foam was aged at $70^{\circ} \mathrm{C}$ under $\mathrm{N}_{2}$ for 1 year in a sealed aluminum canister. A sample of headspace gas was collected into a small gas bottle using a vacuum manifold. An aliquot of a sample gas was admitted to the inlet chamber of a Finnigan 271 mass spectrometer. From the inlet chamber, the gas was allowed to leak slowly into the mass spectrometer's electron-beam ionizer region during an analysis. lons were electrostatically accelerated and mass filtered by an electromagnetic sector to a resolution of $\mathrm{m} / \Delta \mathrm{m} \sim 1400$. The partial pressures of inlet gases that produced the observed ion data were deduced from detector sensitivities. The sum of the molecular gas partial pressures was ratioed to the total measured inlet pressure, and the results were within a few percent of $100 \%$. The NIST mass spectral database was consulted for assignment of hydrocarbons in the headspace analysis.

GPC provided molecular weights and molecular weight distributions of PDMS standards (Aldrich-Sigma) when mixed either with tin octoate or octanoic acid. The GPC instrument consisted of an Alliance 2690 pump equipped with a Wyatt Rex Differential Refractive Index Detector and utilized three Polymer Labs PL Mixed B GPC columns. The solvent used was toluene at $70^{\circ} \mathrm{C}$ and at a flow rate of $1.0 \mathrm{ml} / \mathrm{min}$. The molecular weights were determined using narrow molecular weight PDMS calibration standards.

The foams investigated in this work were aged under compressive load and compression set was used to evaluate aging. Compression set represents the reduction in thickness after a material is aged in compression, and in this work is a fraction of the applied compression. In the experiments described above, S5370 foam samples of initial thickness $H_{i}$ were aged under compressive strain (30\%) for long periods of time. Every 3 months, two specimens per humidity level were removed from the compression fixtures for measurement of their thickness and 
other characterizations. The percent compression set at age $t$ is given by $S=100 *\left(H_{i}-H_{t}\right) /\left(H_{i}-h_{\text {ind }}\right)$, where $\mathrm{S}$ is the compression set, $\mathrm{H}_{\mathrm{i}}$ is the initial thickness, $\mathrm{H}_{\mathrm{t}}$ is the recovered material thickness at age $t, h_{\text {ind }}$ is the height of the indent of the compression fixture. The depth on the compression fixture determines the strain that is applied to the RTV test sample. The thicknesses of the samples were measured 2 hours after compression fixtures were removed from the humidity chambers and the samples were removed from the compression fixtures. In these experiments, a height gauge (Browne and Sharpe Micro-Hite 350) was used to accurately measure the sample thickness.

\section{Results and Discussion.}

\subsection{Artificially Aged SX358 foams.}

SX358 foams were artificially aged under $25 \%$ compressive strain at temperatures varying from 30 to $110^{\circ} \mathrm{C}$ in an open air environment for up to six years. Figure 1 shows that compression set values increased with temperature and aging time. It is important to point out that this behavior is comparable to other data previously reported in the literature for similar foams. For example, Coons and co-workers analyzed the mechanical response of S5370 foams that were aged for 9 years in air with 50\% compressive strain at temperatures varying from 23 to $70^{\circ} \mathrm{C}^{10}$ They assumed that compression set followed a first order kinetic model with Arrhenius temperature dependence. The activation energy was estimated to be approximately $29 \mathrm{~kJ} / \mathrm{mol}$. Patel and co-workers investigated compression set in comparable polysiloxane foams that were aged in dry nitrogen atmospheres at temperatures ranging from 25 to $70^{\circ} \mathrm{C}$ with $25 \%$ compressive strain. ${ }^{11}$ The authors suggested that compression set was not necessarily associated with significant changes in cross-link density, but more likely due to a reorganization of the network. In this work, the role of residues from catalytic active species was investigated in order to explain the compression set behavior observed by our team and others. Specifically, tin(II) residues, which are a product of the foam curing, are of particular interest and are discussed in detail in the next section. 

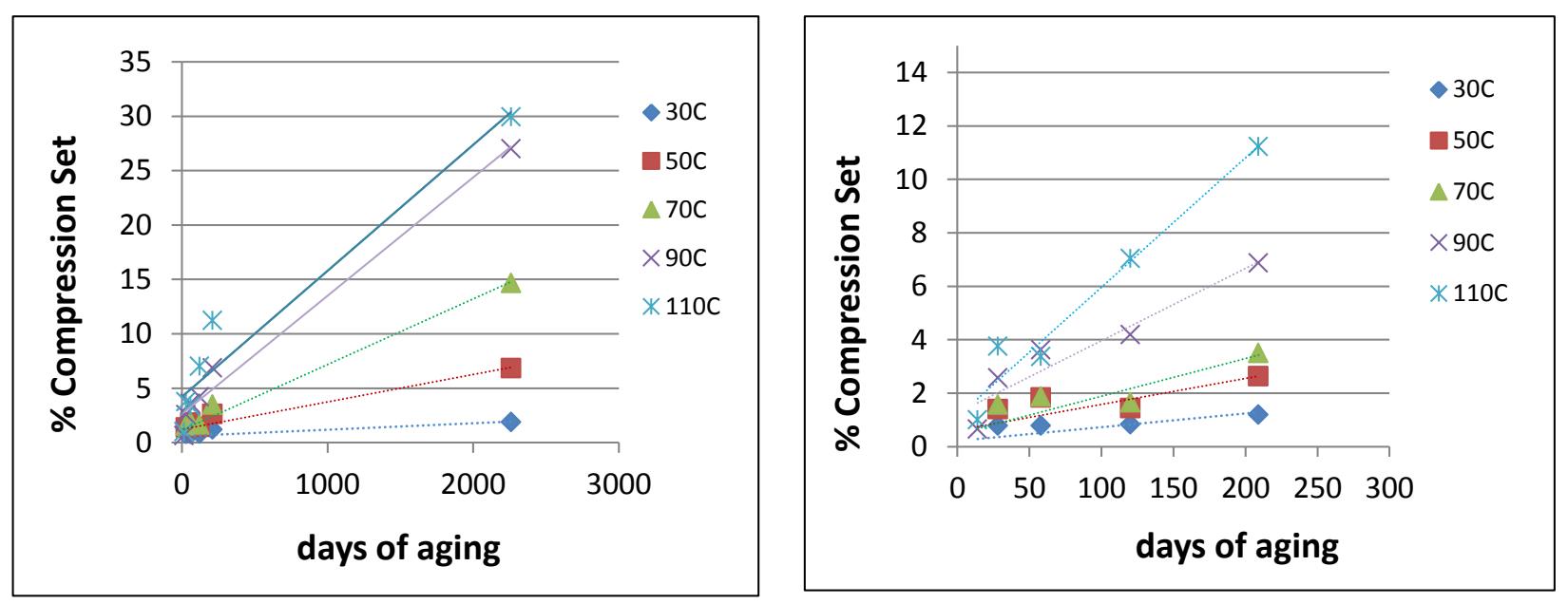

Figure 1. Compression set of artificially aged SX358 foam as a function of aging time: left plot shows data points for up to 6 years, whereas the plot on the right shows data points up to 7 months (the linear fit is just to guide the eye).

\subsection{Oxidation of Tin(II) Octoate Residues.}

RTV polymers are always manufactured by combining a hydroxyl-terminated silicone resin, a cross-linker and a catalyst, with organotin carboxylates as the catalysts of choice since these are less likely to promote side-reactions. ${ }^{16}$ Case in point, the network of SX358 and S5370 foams are made by reactions between dihydroxyl-terminated PDMS chains and crosslinkers (TPS and PMHS). The foams are cured using tin octoate, an effective silanol condensation catalyst routinely used in the silicon industry for curing organosilicon polymers. The curing of SX358 and S5370 foams requires the use of relatively large amounts of tin octoate (5 wt\%), leaving significant amounts of residues in the foam. Interestingly, even though organotin carboxylate catalysts have been used for decades and in a number of applications, the polymerization mechanism is still a subject of consideration. ${ }^{17}$ The work by Severnyi and collaborators provided the first interesting insight on the nature of the active tin center by showing that the silicone curing does not proceed under anhydrous conditions. ${ }^{16}$ This observation led to the work by van der Weij, which suggested that the hydrolysis products are the actual catalysts. ${ }^{18}$ We and others have demonstrated that the tin 
catalyst is prone to oxidation and/or hydrolysis, and these chemical reactions have a direct impact on the catalytic activity and on the final properties of the rubber. ${ }^{15,19}$ In its pristine form, the neat catalyst consists primarily of tin octoate with small amounts of oxidized tin species and octanoic acid. ${ }^{15}$ Distillation of tin octoate does not remove all acid impurities, and since trace amounts of water are always present, these species are readily formed by hydrolysis of the catalyst. $^{20}$ Interestingly, the chemical makeup of tin residues in pristine RTV foams is very different from the composition of the neat tin octoate. Tin residues found in pristine RTV foams are $\operatorname{tin}(\mathrm{II})$ and $\operatorname{tin}(\mathrm{IV})$ species, often in approximately equal concentrations as shown by ${ }^{119} \mathrm{Sn}$ Mössbauer spectroscopy (see Figure 2). Consequently, tin(II) residues remain in the pristine foams, and are likely to promote post-curing reactions amongst residual active groups, such as silanols and silanes. These reactive species are definitely observed in the ${ }^{1} \mathrm{H}$ MAS NMR spectrum of pristine SX358 foams as shown in Figure 3, even though solid-state NMR is not a particularly sensitive technique. The NMR spectrum was truncated to better display the small peaks associated with unreacted $\mathrm{SiH}$ and/or $\mathrm{SiOH}(4.7 \mathrm{ppm})$ and partially unreacted TPS $\left(-\mathrm{OCH}_{2}\right.$ groups at $3.6 \mathrm{ppm})$. The larger peaks in the spectrum are due to methyl protons from PDMS (0.1 ppm), phenyl groups from DPMS (from 7 to $8 \mathrm{ppm}$ ) and residues from the tin catalyst (small peaks from 0.8 to $1.6 \mathrm{ppm}$ ). Post-curing reactions in the presence of these reactive species will likely modify the foam's original network structure by creating new siloxane linkages. The net result will be an increase in the cross-link density. Thus, post-curing reactions are expected to induce changes in the mechanical response of the artificially aged foam. 


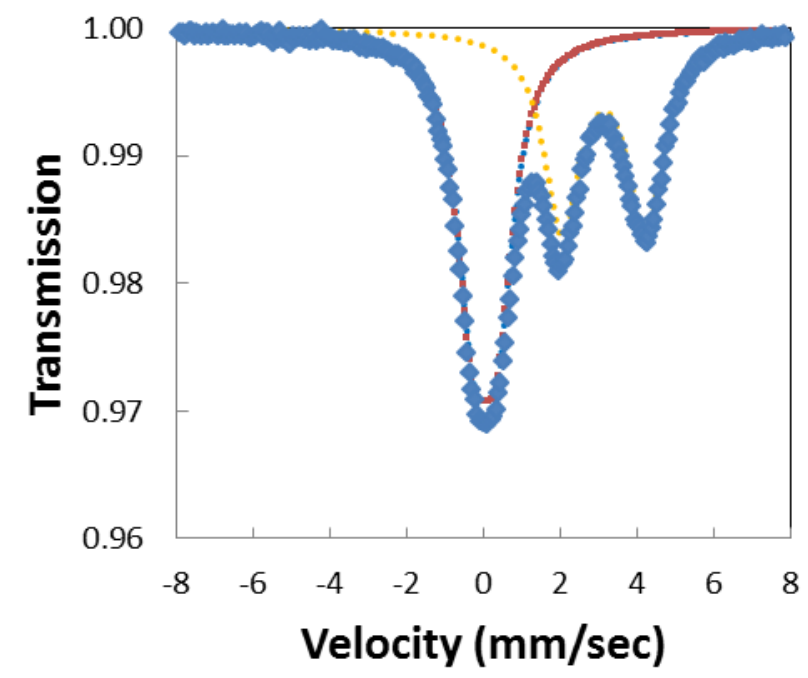

Figure $2 .{ }^{119} \mathrm{Sn}$ Mössbauer spectrum obtained for pristine SX358 foam showing the presence of tin (IV) species (an unresolved doublet centered at $0 \mathrm{~mm} / \mathrm{s}$ ), and tin (II) species (a doublet centered at a Doppler velocity of $3 \mathrm{~mm} / \mathrm{s}$ ). Curve fittings for tin (II) and tin(IV) sites are also shown by dotted lines. Tin relative concentrations are $45 \% \operatorname{tin}($ II) and $55 \% \operatorname{tin}($ IV).

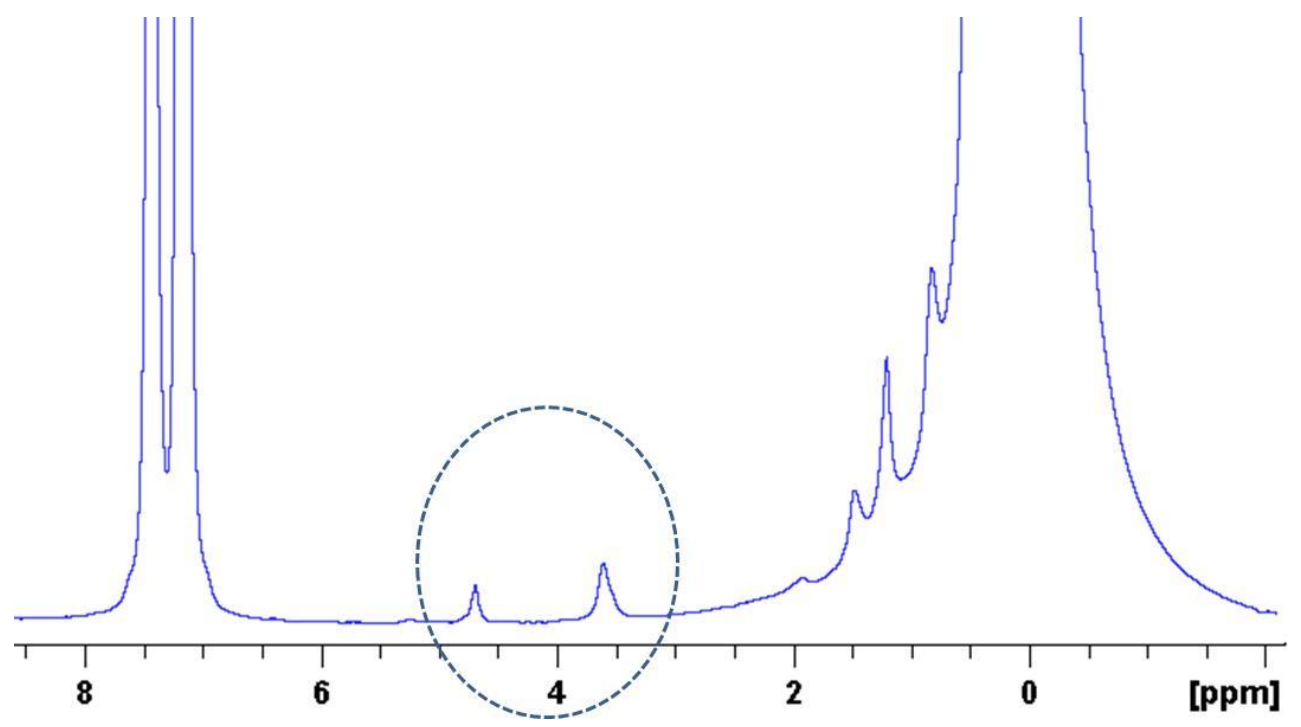

Figure $3 .{ }^{1} \mathrm{H}$ MAS NMR spectrum obtained for pristine SX358 foam showing small peaks in the baseline, which are associated with reactive species such as silanols and silane groups. 
Compelling evidence showing that post-curing reactions actually take place during aging of the SX358 foam is provided by analysis of the evolved gases. A SX358 sample that was aged in a sealed container under $\mathrm{N}_{2}$ afforded the means to evaluate permanent gases and volatiles generated due to thermal aging. The SX358 sample was artificially aged at $70^{\circ} \mathrm{C}$ for a period of 1 year. As listed in Table 1, characterization of the headspace by mass spectroscopy clearly shows $\mathrm{H}_{2}$ evolution. This gas is a by-product of condensation reactions between silanol and silane groups. It is worth pointing out that such chemical reactions are promoted by tin octoate, and will lead to new Si-O-Si linkages. Clearly, this result indicates that tin(II) residues are catalytically active and able to promote post-curing reactions of reactive species, which in turn may lead to compression set. Cyclic siloxanes are also detected in the headspace, albeit in very small concentrations. These species could be formed through back-biting reactions promoted by residues of the tin catalyst.

Table 1. Major constituents detected in the headspace of a canister containing $12 \mathrm{~g}$ of SX358 foam aged under inert conditions at $70^{\circ} \mathrm{C}$ for 1 year. Concentrations are in ppm. D3 and D4 refer to hexamethylcyclotrisiloxane and octamethylcyclotetrasiloxane, respectively. A control sample shows only $22 \mathrm{ppm} \mathrm{H}_{2}$.

\begin{tabular}{|c|c|c|c|c|c|c|c|}
\hline $\mathrm{H}_{2}$ & $\mathrm{CH}_{4}$ & $\mathrm{Ar}$ & $\mathrm{CO}_{2}$ & $\mathrm{C}_{3} \mathrm{H}_{8} \mathrm{O}$ & $\mathrm{C}_{6} \mathrm{H}_{6}$ & $\mathrm{D} 3$ & $\mathrm{D} 4$ \\
\hline 3,000 & 10.8 & 182 & 144 & 1.4 & 8.6 & 26.7 & 0.7 \\
\hline
\end{tabular}

Since tin octoate is prone to oxidation reactions, the question arises as to when tin(II) residues become completely oxidized. Changes in the oxidation state of tin(II) residues in artificially aged SX358 foams were investigated by ${ }^{119}$ Sn Mössbauer spectroscopy. It was found that higher aging temperatures and longer aging times resulted in an increase of the relative concentration of tin(IV) species, and likewise a decrease in the concentration of tin (II) species. Figure 4 illustrates the change in the tin(IV) relative concentration as a function of time. This 
master curve was obtained by using the time-temperature superposition (TTS) approach. ${ }^{21}$ The activation energy for the tin(II) oxidation process was found to be approximately $50 \mathrm{~kJ} / \mathrm{mol}$.
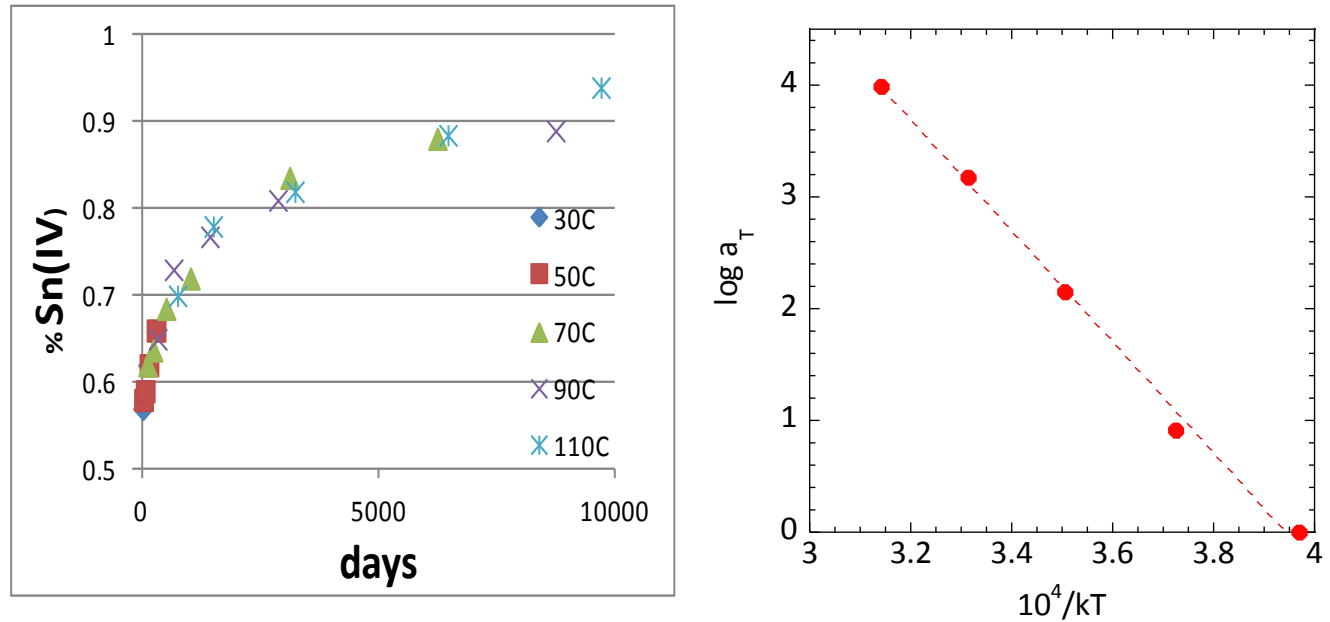

Figure 4. Increase in the relative concentration of $\operatorname{tin}(\mathrm{IV})$ species due to oxidation reactions as a function of time and temperature (left) and TTS shift factors (right).

Based on these results, it was of interest to investigate procedures that could rapidly oxidize tin(II) residues. One of the most effective methods found by our team consisted of treating the pristine foam with $10 \%$ hydrogen peroxide solution for up to 30 minutes, followed by washing in water and drying in air. The final treatment consisted of post-curing the "peroxide washed" foam at $115^{\circ} \mathrm{C}$ in air for about 3 hours. ${ }^{119} \mathrm{Sn}$ Mössbauer data obtained for samples treated by this method show no tin(II) species after the post-curing treatment (see Figure 5). Furthermore, ${ }^{29} \mathrm{Si}$ MAS NMR spectrum of the treated foam showed no detectable damage to the polymer. Another method for oxidizing tin(II) residues consisted of exposing the foam to gamma irradiation in air. However, this approach has some limitations since the RTV foam is susceptible to radiolysis. ${ }^{22}$ 

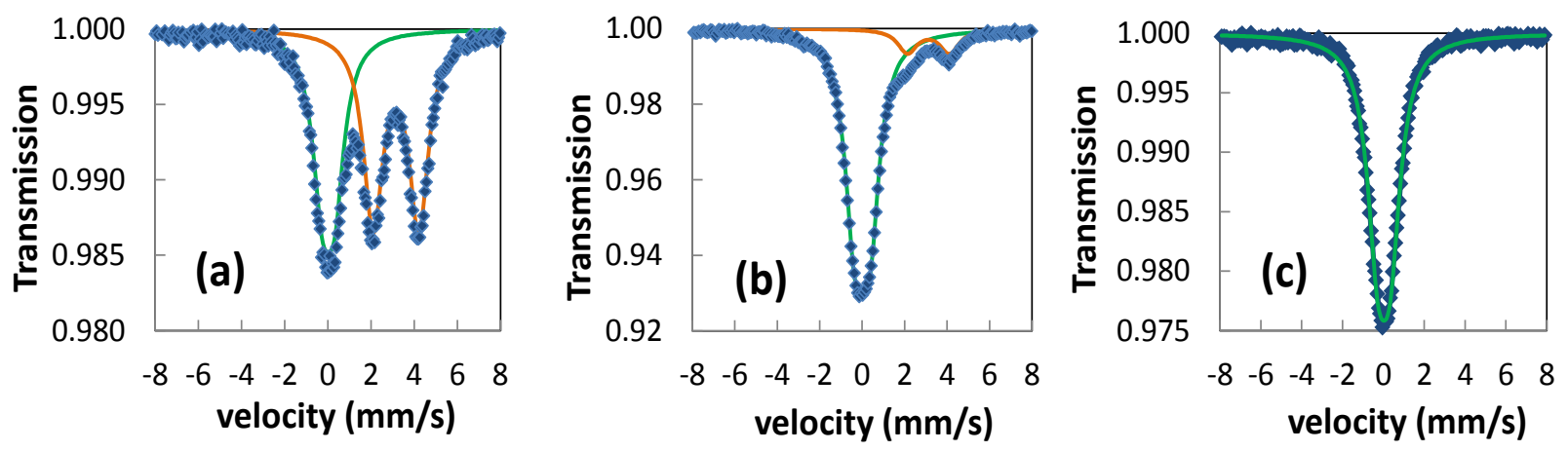

Figure 5. Evolution of the oxidation state of tin residues as the RTV foam receives different treatments: (a) pristine foam (non-post-cured) has 60\% tin(II) species, (b) pristine foam treated with hydrogen peroxide has $12 \% \operatorname{tin}(\mathrm{II})$ species, and (c) foam treated with hydrogen peroxide and then post-cured has no tin(II) species, only tin(IV) species. Curve fittings of tin(IV) species (green trace), and tin(II) species (red trace) are represented as well.

\subsection{Artificially aged $S 5370$ foams.}

This work demonstrated that specific residues associated with the tin catalyst promote post-curing reactions that, in the long term, could be detrimental to the life of the foam and lead to mechanical failure. Once tin(II) residues are oxidized, other aging mechanisms may still take place and influence the mechanical performance of the RTV foam. Thus, S5370 foams that were already naturally aged for 28 years under standard shelf storage and never used in service were chosen for the second part of this aging study. ${ }^{119}$ Sn Mössbauer experiments confirmed that S5370 foams had very small amounts of tin(II) residues, namely about $12 \%$ on average. As shown in Figure 6 , by the end of the aging study the foams had only 4 to $6 \%$ tin(II) species (the remaining being tin(IV) species). 


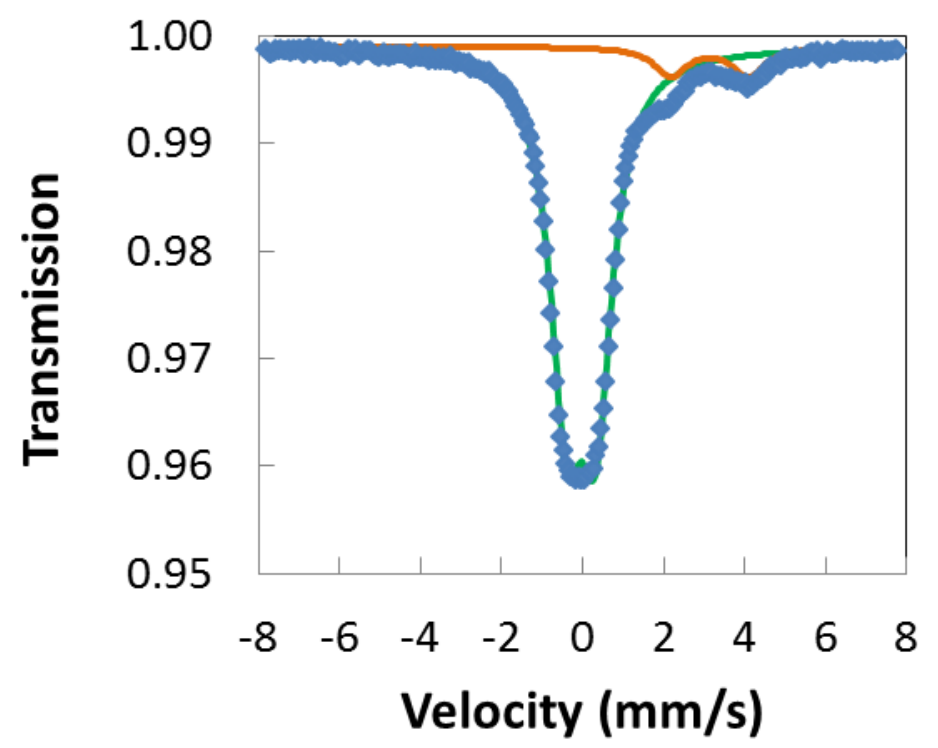

Figure 6. ${ }^{119} \mathrm{Sn}$ Mössbauer spectrum obtained for 28 year-old $\mathbf{S} 5370$ foam that was aged for 21 months at high humidity showing that most tin(II) residues are oxidized. There are only $6 \%$ Sn(II) species. Curve fittings of tin(IV) species (green trace), and tin(II) species (red trace) species are represented as well. No significant differences in the tin (II) oxidation rate were observed with humidity levels.

In addition to tin(II) residues, it was of interest to investigate other non-network species that reside in the 28 year-old foam. The soluble fraction not only has a plasticizer effect, but it may also contribute to the aging of the foam. Analysis of the extractable material obtained from $\mathrm{S} 5370$ foams shows the presence of octanoic acid as detected by liquid-state ${ }^{1} \mathrm{H}$ NMR. This acid is produced when tin octoate is exposed to trace amounts of moisture. Figure 7 shows that extracts consist mostly of a large peak corresponding to methyl groups from PDMS chains, and smaller peaks that are associated with organic residues from the tin catalyst and octanoic acid (1.085 to $0.75 \mathrm{ppm}$ are methyl groups, 1.2 to $1.6 \mathrm{ppm}$ are due to methylene protons, and peaks from 2.2 to $2.3 \mathrm{ppm}$ are methyne protons). Residues from unreacted DPMS and possibly by- 
products like dimers are also present in the extracts; aromatic protons show up as a set of peaks at lower field (7.2 to $7.6 \mathrm{ppm}$ ), whereas the methyl protons appear at 0.5 to $0.7 \mathrm{ppm}$.

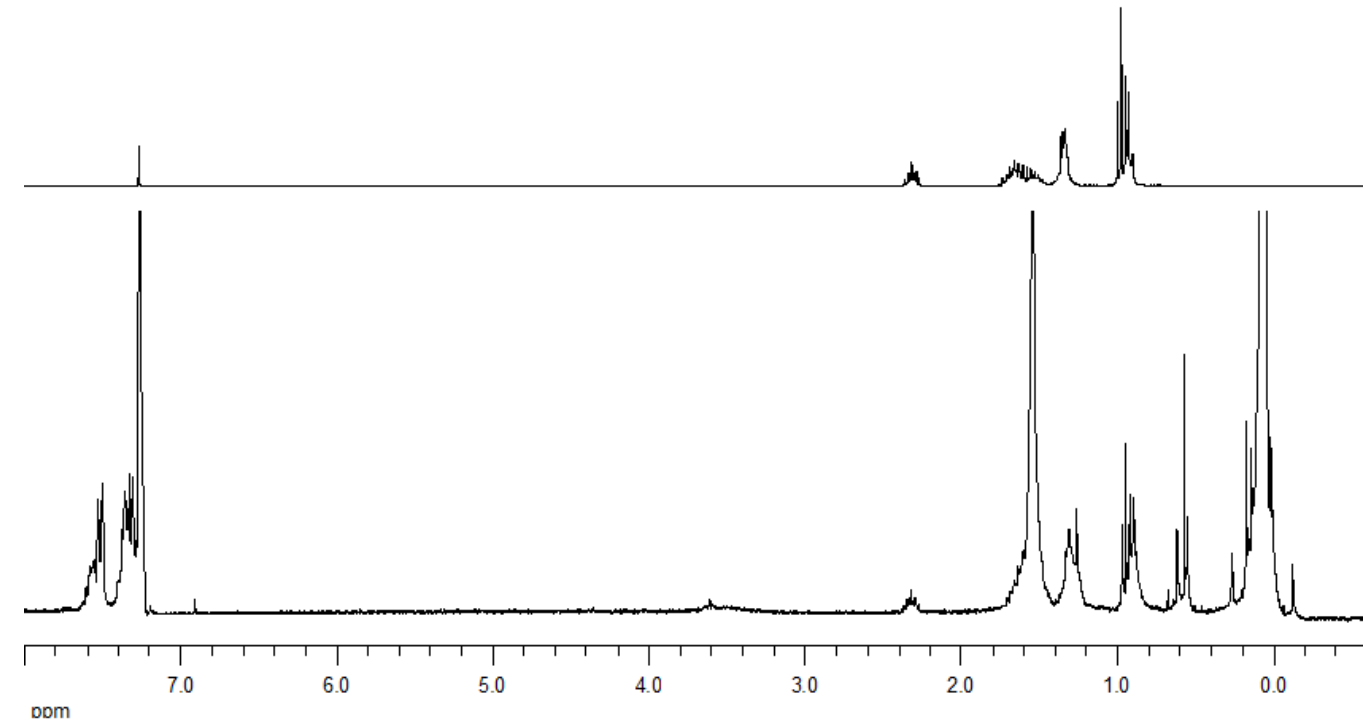

Figure 7. ${ }^{1} \mathrm{H}$ NMR spectrum of extractable material obtained from S5370 foams (bottom). The sharp peak at $7.26 \mathrm{ppm}$ is due to residual protons from $\mathrm{CDCl}_{3}$, the solvent used to extract the foam, and the peak at $1.56 \mathrm{ppm}$ is due to residual water. The top ${ }^{1} \mathrm{H}$ NMR spectrum is of neat octanoic acid dissolved in $\mathrm{CDCl}_{3}$.

S5370 foams were artificially aged under compression at $70^{\circ} \mathrm{C}$ for up to two years. These specimens were aged in high humidity conditions in order to probe the effects of environments other than air. The reasoning for choosing this set of aging conditions was to probe the combined effect of octanoic acid residues and moisture on the degradation of the foam. Thus, S5370 foams afforded the opportunity to gauge the detrimental effects of other reactive species other than tin(II) residues, since these were mostly oxidized in the 28 year-old foam. It has been previously suggested that residual octanoic acid contributes to the long term compression set observed in S5370 foams, and that improved post-curing procedures (longer 
post-curing times under light vacuum) may reduce their concentration. ${ }^{23}$ Figure 8 shows the averaged compression set observed for $\mathbf{S} 5370$ foams that were thermally aged under compressive load (30\%) in presence of moisture. It is clear that compression set increases linearly with aging, and it reaches very high values for the foams aged for the longest amount of time. Compression set is also more pronounced for the samples aged under higher humidity levels than for those aged at lower humidity levels. These results indicate that, even in the absence of tin(II) residues, $\mathbf{S} 5370$ foams continue to age when exposed to high humidity environments. In order to elucidate such compression set behavior, the catalytic activity of species originated from the degradation of tin octoate residues was evaluated.

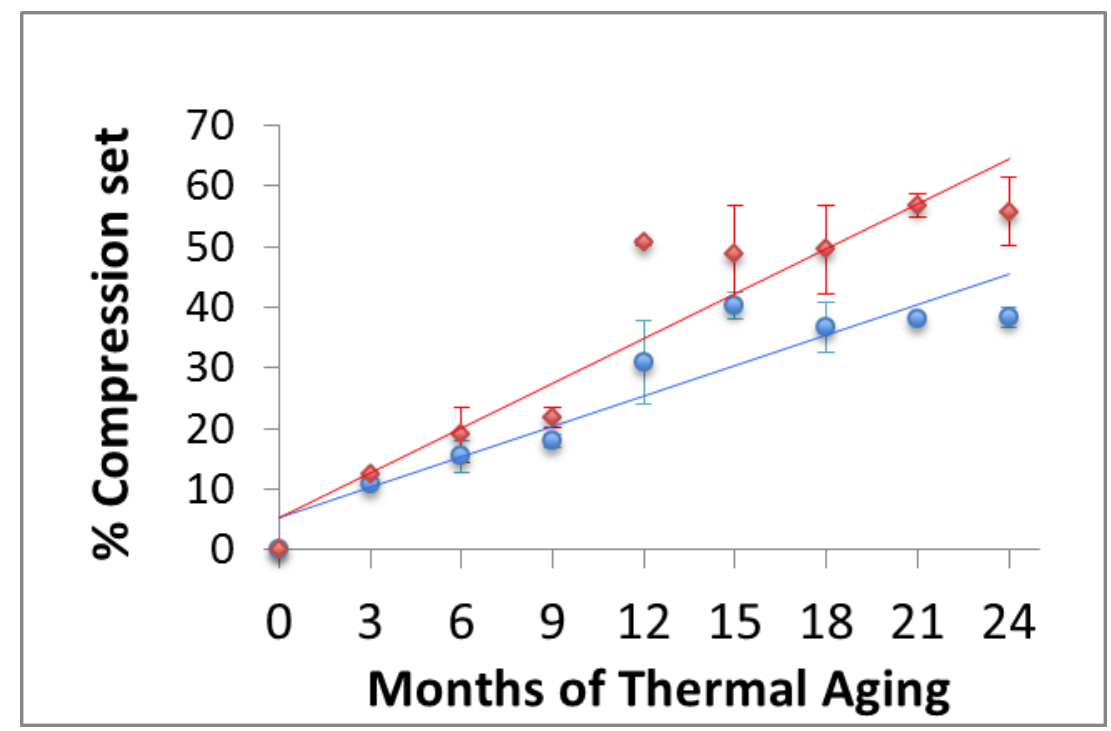

Figure 8. Compression set of $\mathrm{S} 5370$ samples aged at $70^{\circ} \mathrm{C}$ at 10 (blue trace) and $25 \% \mathrm{RH}$ (red trace) for up to 2 years.

\subsection{Catalytic Activity of Tin Residues.}

As previously mentioned, tin octoate slowly oxidizes producing tin(IV) species and undergoes hydrolysis producing octanoic acid. ${ }^{15,24,25}$ The presence of the acid in the RTV foam may lead to the polymer degradation by protonation of the siloxane chain, which eases the 
barrier for hydrolysis. ${ }^{26}$ In order to demonstrate this effect, trimethyl-terminated polydimethylsiloxane samples were thermally aged in presence of octanoic acid and trace amounts of water. These samples were mixed with $5 \mathrm{wt} \%$ octanoic acid and aged at 40, 70, 90 and $110^{\circ} \mathrm{C}$ for various periods of time (up to 103 days for the $40^{\circ} \mathrm{C}$ sample, less for the other samples). Changes in the molecular weight of the artificially aged PDMS samples were periodically assessed by GPC measurements. A significant drop in the molecular weight was observed as shown in Figure 9. The activation energy for the hydrolysis reaction, promoted by trace amounts of moisture and carboxylic acid, was estimated by the time-temperature superposition approach (TTS). It was found to be approximately $30 \mathrm{~kJ} / \mathrm{mol}$, which is consistent with results obtained by other authors for polydimethylsiloxane networks. $7,8,27,28$
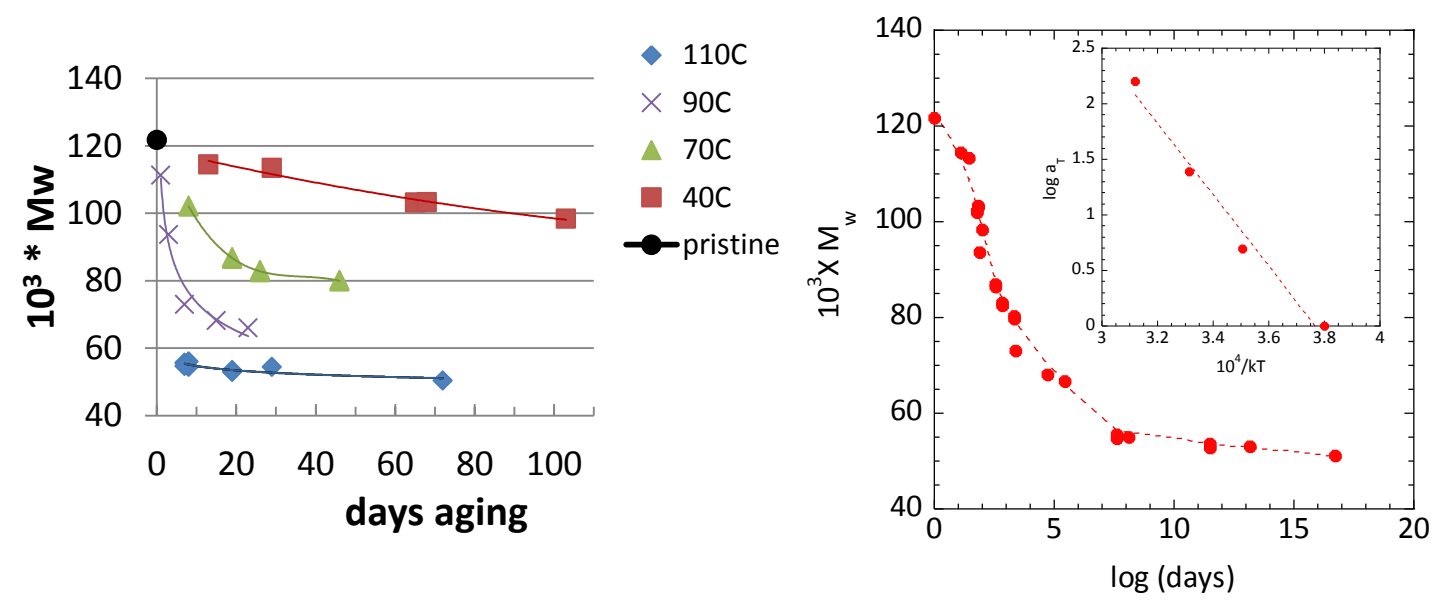

Figure 9. Decrease in the average molecular weight of linear PDMS when thermally aged with octanoic acid (left), and TTS master curve (right) with shift factors (insert).

Residues from the tin catalyst are also thought to promote bond-exchange reactions, which are rearrangements of the network (basically an active unit attaches to an existing unit by replacing a pre-existing bond with a new one). More specifically, trace amounts of water and 
acidic species (such as octanoic acid) can attack the polar O-Si-O backbone and induce chain scission with formation of silanols. The tin catalyst then promotes the condensation of the newly formed silanol groups, resulting in a network with a broader molecular weight distribution than the original one. This bond-exchange effect was investigated by monitoring the molecular weight distribution of tri-methyl-terminated PDMS samples that were mixed with the tin catalyst. Significant changes in the molecular weight distribution were observed for the aged samples when compared with the control sample, as seen in Figure 10. The polydispersity of the 10,000 Dalton sample changed from 1.2 to 1.6 and 1.9 after being thermally aged for 3 and 6 weeks at $70^{\circ} \mathrm{C}$; respectively.

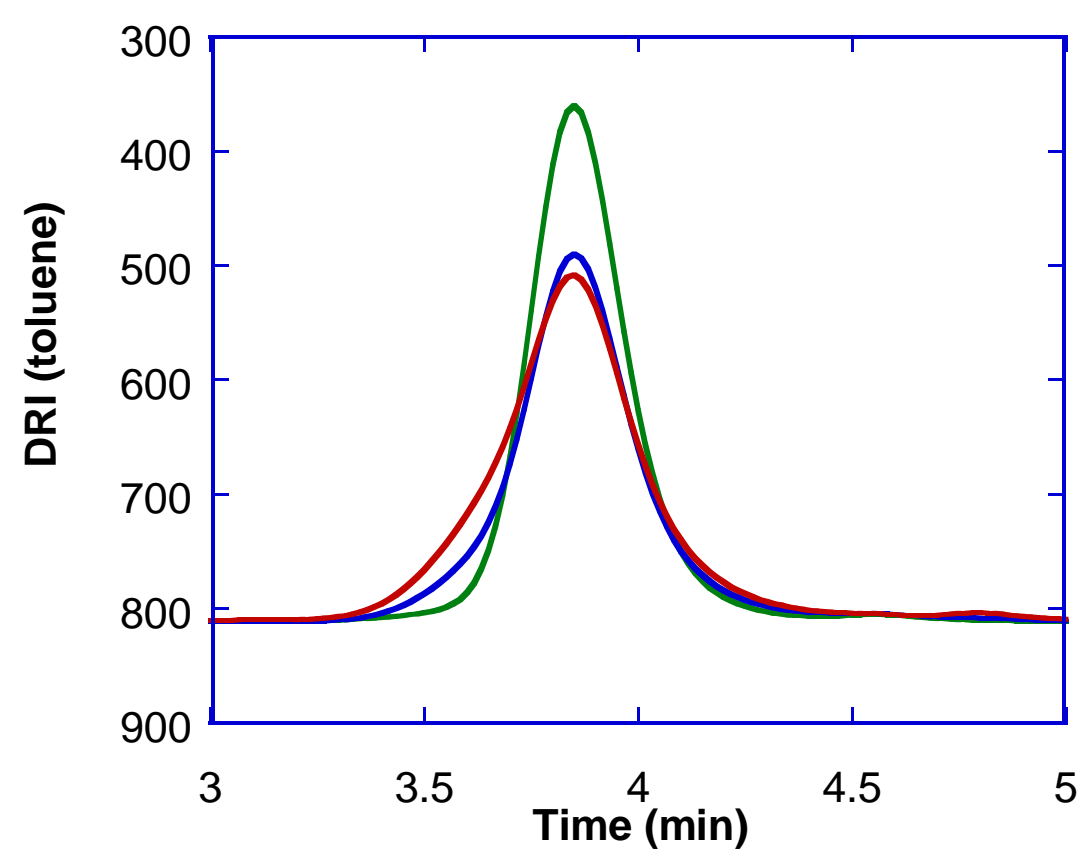

Figure 10. GPC chromatograms obtained for the 10,000 Dalton tri-methyl terminated PDMS samples after being aged with $5 \mathrm{wt} \%$ tin octoate for 3 weeks at $70^{\circ} \mathrm{C}$ (blue trace), and the same sample after aged for 6 weeks at $70^{\circ} \mathrm{C}$ (red trace). The green trace is the control sample. (DRI stands for the differential refractometer index). 
Determining if bond-exchange reactions could take place in RTV foams was of interest as well. Thus, the experiment performed here consisted of artificially aging SX358 foams at $60^{\circ} \mathrm{C}$ for up to 23 weeks with excess of isotopically enriched $\mathrm{H}_{2}{ }^{17} \mathrm{O}$. The ${ }^{17} \mathrm{O}$ NMR signal was used as a signature to probe the insertion of ${ }^{17} \mathrm{O}$ in the polymer chain. Two sets of foams were aged by this procedure: foams that had been post-cured and those non-post-cured. NMR results obtained for these artificially aged samples are shown in Figure 11. The large peak at 0.0 ppm is associated with ${ }^{17} \mathrm{O}$ signal from free water, whereas the peak at $71.2 \mathrm{ppm}$ is due to $-\mathrm{Si}^{-17} \mathrm{O}-\mathrm{Si}$ groups, confirming the proposed chemical reaction described in Figure $11 .{ }^{29,30}$ It is interesting to note that the non-post-cured sample also shows a peak at $43.9 \mathrm{ppm}$, which we assume to be associated with $-\mathrm{Si}_{-}{ }^{17} \mathrm{OH}$ groups. The presence of silanol groups in the non-post-cured foam is not unexpected, since this foam is likely to contain relatively large amounts of unreacted silanols from the dihydroxyl-terminated PDMS chains. These reactive groups may have exchanged with $\mathrm{H}_{2}{ }^{17} \mathrm{O}$, producing the small peak observed in the NMR spectrum.

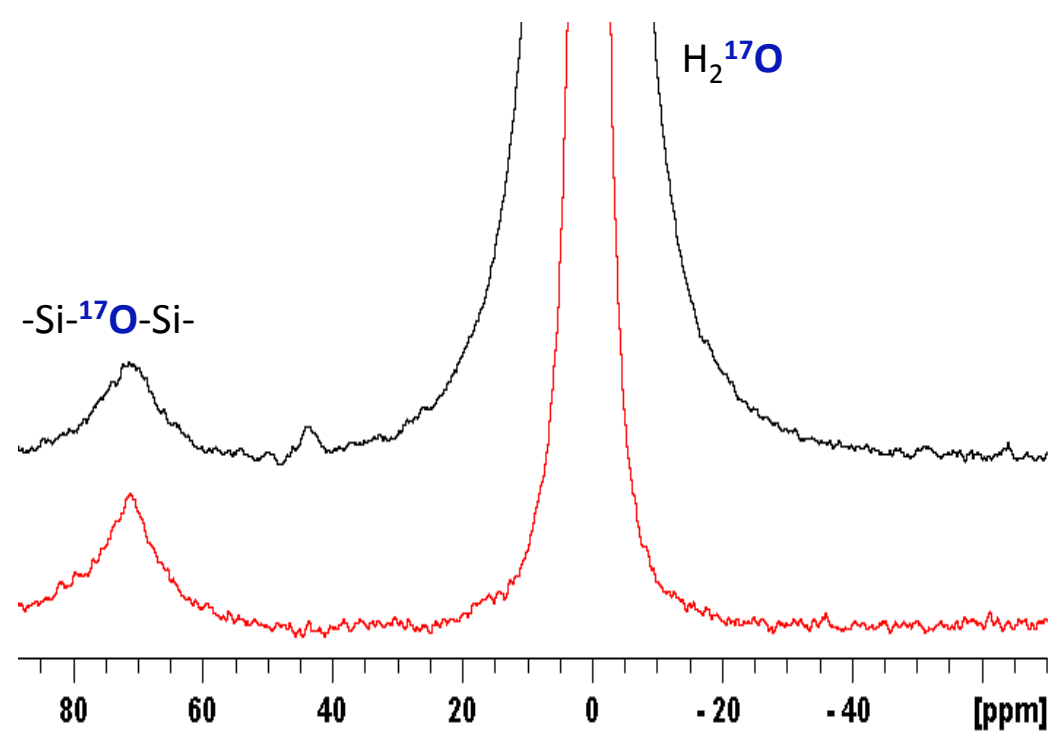




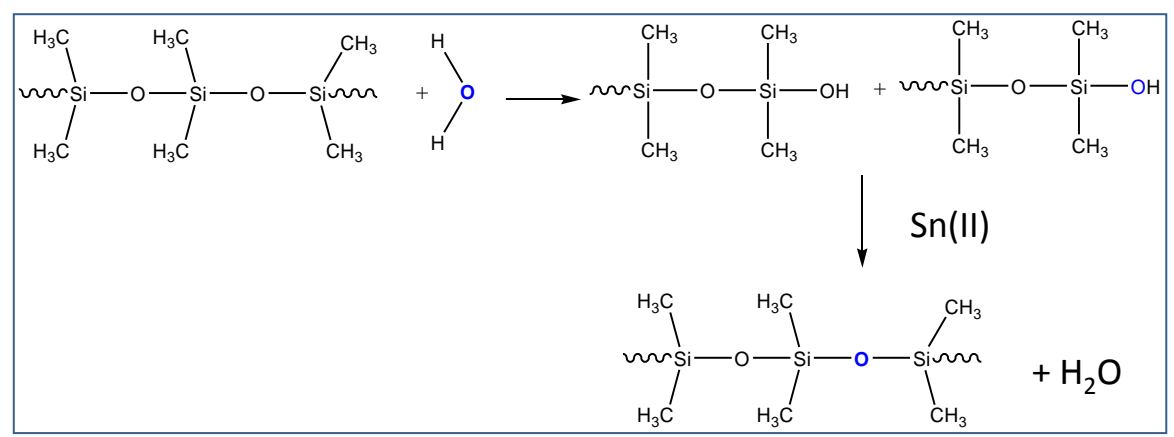

Figure 11. ${ }^{17} \mathrm{O}$ MAS NMR spectra for post-cured (bottom) and non-post-cured (top) SX358 foams that were thermally aged with ${ }^{17} \mathrm{O}$ isotopically enriched water. The scheme for the ${ }^{17} \mathrm{O}$ insertion in the polymer backbone is shown as well (O represents $\left.{ }^{17} \mathrm{O}\right)$. The condensation of silanol groups is promoted by the tin catalyst.

It was previously established that even in the absence of a cross-linker, tin octoate is able to catalyze chain extension reactions of dihydroxyl-terminated PDMS. ${ }^{31-33}$ The catalytic activity of oxidized tin residues was then assessed by combining low molecular weight dihydroxyl-terminated PDMS with two different batches of tin octoate catalyst: one obtained from an unused bottle and a second one from an opened bottle that had degraded naturally. Both samples were initially characterized by ${ }^{119} \mathrm{Sn}$ Mössbauer spectroscopy to determine the relative concentration of $\operatorname{tin}(\mathrm{II})$ and $\operatorname{tin}(\mathrm{IV})$ species. The pristine sample contained about $16 \%$ $\operatorname{tin}($ IV) and $84 \% \operatorname{tin}(I I)$, whereas the degraded material contained $71 \% \operatorname{tin}($ IV) and $29 \% \operatorname{tin}(I I)$. Once the dihydroxyl-terminated PDMS samples were mixed with one of these two catalysts, the resulting silanol condensation reactions were followed by liquid-state ${ }^{29} \mathrm{Si} N \mathrm{NR}$ spectroscopy. ${ }^{29} \mathrm{Si}$ NMR is well suited for probing this type of reaction, since the peak associated with end groups, i.e, HO-Si groups (at -11.1 ppm), is clearly distinguishable from peaks associated with silicons in the polymer backbone, -O-Si-O- (from -21 to $-22.5 \mathrm{ppm}$ ). Since the dihydroxylterminated PDMS used in this experiment has a low averaged molecular weight, silicons have distinct chemical shifts depending on their proximity to the end groups. Figure 12 shows ${ }^{29} \mathrm{Si}$ NMR spectra obtained for the PDMS samples that contained the pristine and naturally aged tin 
octoate. This spectrum is exactly like the one for the neat polymer. As can be seen, the tin catalyst with a high tin(IV) content does not promote the condensation chemistry, whereas the sample with a very low tin(IV) content promptly catalyzes the reaction. This is verified by the disappearance of the Si-OH groups for the PDMS sample that had the catalyst with low tin(IV) content.

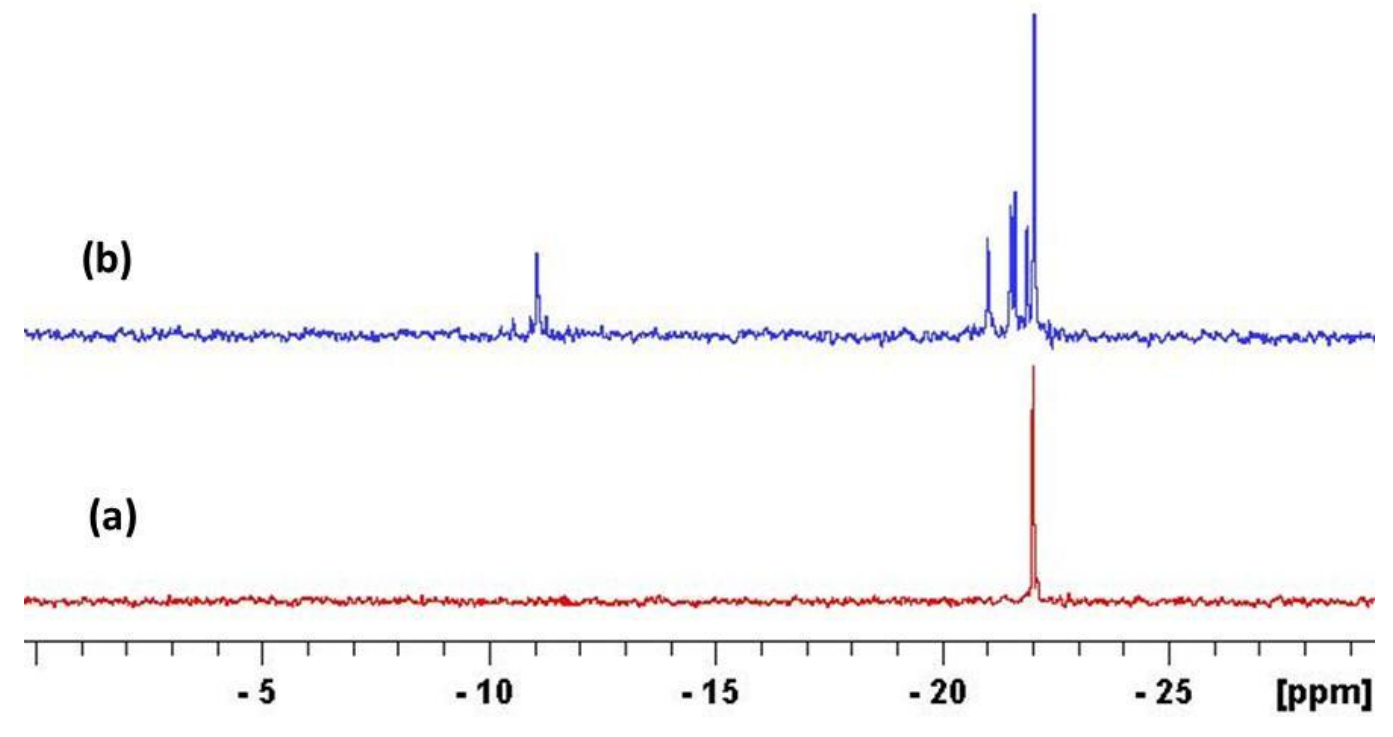

Figure 12. ${ }^{29}$ Si NMR spectra obtained for dihydroxyl-terminated PDMS mixed with tin octoate containing either high or low tin(IV) concentrations. (a) PDMS sample mixed with tin octoate containing $18 \%$ tin(IV) species, and (b) PDMS sample mixed with tin octoate containing $71 \%$ of tin (IV) species. This spectrum is exactly as the one of the neat polymer. NMR experiments were performed at room temperature.

\section{Conclusions.}

The goal of the present work was to identify aging mechanisms that degrade RTV polysiloxane foams when subjected to aging under compressive strain. Two sets of foams were artificially aged either in high humidity environments or ambient conditions. It was found that 
compression set was more pronounced in foams aged in presence of moisture than in foams aged in an open air environment. Since the RTV foams are synthesized using relatively large amounts of a tin(II) catalyst, it was important to identify chemical reactions that could be catalyzed by its residues. The degradation of the tin catalyst, which occurs through oxidation and hydrolysis processes, produces tin(IV) species and hexanoic acid. Our results showed that a degraded catalyst sample that contained a large ratio of tin(IV) to tin(II) species no longer readily catalyzed silanol condensation reactions in dihydroxyl-terminated PDMS, indicating loss of catalytic activity with oxidation. The carboxylic acid, once combined with trace amounts of water, was found to promote chain scission reactions in trimethyl-terminated PDMS as demonstrated by GPC experiments. Furthermore, tin residues combined with trace amounts of water were able to catalyze bond-exchange reactions in trimethyl-terminated PDMS. The same reaction was also observed by ${ }^{17} \mathrm{O} N M R$ in $\mathrm{RTV}$ foams thermally aged with excess of ${ }^{17} \mathrm{O}$ isotopically enriched water. Thus, chain scission and bond-exchange reactions were identified as the main reactions promoted by trace amounts of water when combined with tin residues and octanoic acid. Based on these observations, S5370 foams containing mainly oxidized tin catalyst were artificially aged at $70^{\circ} \mathrm{C}$ under compressive load, and in high humidity environments. These foams were 28 years-old, and were chosen because of their low tin(II) residue concentration. It was determined that accelerated aged S5370 foams exhibited large compression set, which was attributed to chemical relaxation of the polymer chain largely induced by chain scission reactions. On the other hand, SX358 foams aged under lab conditions showed compression set that was mostly attributed to post-curing reactions. These were catalyzed by $\operatorname{tin}(\mathrm{II})$ residues and accompanied by $\mathrm{H}_{2}$ evolution. In conclusion, the picture that emerged is a detrimental one regarding the long-term use of RTV foams under compressive strain and in high humidity environments.

Acknowledgments: This work was funded by the Enhanced Surveillance Campaign, and the US Department of Energy's National Nuclear Security Administration under contract DEAC52-06NA25396. 


\section{References.}

(1) Mark, J. E. Accounts Chem Res 2004, 37, 946.

(2) Grassie, N.; Macfarlane, I. G. Eur Polym J 1978, 14, 875.

(3) Grassie, N.; Francey, K. F.; Macfarlane, I. G. Polym Degrad Stab 1980, 2, 67.

(4) Grassie, N.; Francey, K. F. Polym Degrad Stab 1980, 2, 53.

(5) Visser, S. A.; Hewitt, C. E.; Binga, T. D. J Polym Sci Pol Phys 1996, 34, 1679.

(6) Thomas, T. H.; Kendrick, T. C. J Polym Sci A2 1969, 7, 537.

(7) Stein, J.; Prutzman, L. C. J Appl Polym Sci 1988, 36, 511.

(8) Osthoff, R. C.; Bueche, A. M.; Grubb, W. T. J Am Chem Soc 1954, 76, 4659.

(9) Lin, S. B. Acs Sym Ser 1995, 603, 37.

(10) Coons, J. E.; McKay, M. D.; Hamada, M. S. Polym Degrad Stab 2006, 91, 1824.

(11) Patel, M.; Chinn, S.; Maxwell, R. S.; Wilson, T. S.; Birdsell, S. A. Polym Degrad Stab 2010,

95, 2499.

(12) Gilbert, A. R.; Kantor, S. W. J Polym Sci 1959, 40, 35.

(13) Andrews, R. D.; Tobolsky, A. V.; Hanson, E. E. J Appl Phys 1946, 17, 352.

(14) Rottach, D. R.; Curro, J. G.; Grest, G. S.; Thompson, A. P. Macromolecules 2004, 37, 5468.

(15) Labouriau, A.; Taylor, D.; Stephens, T. S.; Pasternak, M. Polym Degrad Stab 2006, 91,

1896.

(16) Severnyi, V. V.; Minasyan, R. M.; Makarenko, I. A.; Bizyukova, N. M. Vysokomol Soedin $a+1976,18,1276$.

(17) Cervantes, J.; Zarraga, R.; Salazar-Hernandez, C. Appl Organomet Chem 2012, 26, 157.

(18) Vanderweij, F. W. Makromol Chem 1980, 181, 2541.

(19) Cypryk, M.; Apeloig, Y. Organometallics 2002, 21, 2165.

(20) Leenslag, J. W.; Pennings, A. J. Makromol Chem 1987, 188, 1809.

(21) Ferry, J. D. Viscoelastic properties of polymers; Wiley: New York, 1980.

(22) Labouriau, A.; Cady, C.; Gill, J.; Taylor, D.; Zocco, A.; Stull, J.; Henderson, K.; Wrobleski, D. Polym Degrad Stab 2015, 117, 75.

(23) Robinson, M. W. C.; Swain, A. C.; Khan, N. A. Polym Degrad Stab 2015, 116, 88.

(24) Blair, M. W.; Muenchausen, R. E.; Taylor, R. D.; Labouriau, A.; Cooke, D. W.; Stephens, T. S. Polym Degrad Stab 2008, 93, 1585.

(25) Hubert, C.; Ziemons, E.; Rozet, E.; Breuer, A.; Lambert, A.; Jasselette, C.; De Bleye, C.; Lejeune, R.; Hubert, P. Talanta 2010, 80, 1413.

(26) Lai, S. K.; Batra, A.; Cohen, C. Polymer 2005, 46, 4204.

(27) Kantor, S. W.; Grubb, W. T.; Osthoff, R. C. J Am Chem Soc 1954, 76, 5190.

(28) Johnson, D. H.; Mcloughlin, J. R.; Tobolsky, A. V. J Phys Chem-Us 1954, 58, 1073.

(29) Alam, T. M. Rad Phys Chem 2001, 62, 145.

(30) Patel, M.; Morrell, P.; Cunningham, J.; Khan, N.; Maxwell, R. S.; Chinn, S. C. Polym Degrad Stab 2008, 93, 513.

(31) He, X. W.; Widmaier, J. M.; Herz, J. E.; Meyer, G. C. Eur Polym J 1988, 24, 1145.

(32) Normand, F.; He, X. W.; Widmaier, J. M.; Meyer, G. C.; Herz, J. E. Eur Polym J 1989, 25,

371.

(33) Clarson, S. J.; Wang, Z. M.; Mark, J. E. Eur Polym J 1990, 26, 621. 